registered in the Danish Hip or the Danish Knee Arthroplasty Registers (DHA/DKA) with a diagnosis of primary OA were sent a detailed questionnaire regarding previous occupation, related exposures and complementary environmental factors. The analyses included cumulated exposures, McNemar`s $X^{2}$ tests, and conditional logistic regression including gene-exposure-interaction variables.

Results 1181 twins responded (rate 58.9\%). Responder analyses did not display any significant difference with non-responders with respect to diagnosis, zygosity and sex. We found a gene-exposure effect modification in hip OA-lifting and lifting-walking with OR`s $17.7(1.1-280.2)$ and 10.4 (1.00107.1), respectively, and a clear dose-response relationship between hip OA and prolonged standing-walking. Significant occupational risk factor in knee OA was kneeling, but no gene-kneeling interaction was detectable.

Conclusion Gene-exposure effect modification may be important in the development of hip OA in particular exposures to lifting and lifting-walking, but not in knee OA.

\section{A COMPARATIVE STUDY OF MUSCULOSKELETAL SYMPTOMS AND WORK- OR STUDY-RELATED IMPACT FOR PROFESSIONAL AND PRE-PROFESSIONAL MUSICIANS}

Jessica Stanhope*, Philip Weinstein, Rebecca Tooher, Dino Pisaniello. The University of Adelaide, Adelaide, Australia

\subsection{6/oemed-2018-ICOHabstracts.749}

Introduction Musculoskeletal symptoms are common in musicians, but little is known of the work- or study-related impacts, nor how they compare with other groups. The aim of this study was to compare professional musicians and preprofessional musicians (university music students), with a reference group, regarding the prevalence of musculoskeletal symptoms and their impact.

Methods A questionnaire survey was distributed to university music students and professional musicians, as well as nonmusic university staff and students (the reference group). Ache, pain and discomfort in the previous 12 months were determined using a modified Nordic Musculoskeletal Questionnaire, as well as the work- and study-related impact of these symptoms. Descriptive statistics were reported, and comparisons were made adjusting for age and gender. A 5\% level of significance was used.

Result Symptom prevalence was high in both groups $(86 \%$ for musicians and $91 \%$ for the reference group), principally in the neck, shoulder and lower back regions. After adjusting for age and gender, symptoms in the wrist/hand region were more common for musicians (OR 1.55, 95\% CI: 1.12 to 2.15 ), and less common in the lower back (OR 0.69, 95\% CI: 0.50 to 0.95), hip/thigh (OR $0.45,95 \% \mathrm{CI}: 0.31$ to 0.68 ), knee (OR $0.45,95 \% \mathrm{CI}: 0.31$ to 0.66 ), and ankle/foot (OR 0.40, 95\% CI: 0.27 to 0.58 ) when compared with the reference group.

Musicians were more likely to make changes to their work or study (OR 2.08, 95\% CI: 1.27 to 3.39), or take leave from work or study (OR 1.71, 95\% CI: 1.12 to 2.60) because of their musculoskeletal symptoms, when compared with the reference group.

Discussion Musculoskeletal symptoms were common in both groups, with musicians more likely to experience wrist/hand symptoms. Musicians' were more likely to experience an impact from musculoskeletal symptoms on their work or study. Implications will be discussed.

\section{ASSOCIATION BETWEEN KINESIOPHOBIA AND PRESENTEEISM AMONG ELDERCARE WORKERS WITH LBP}

${ }^{1}$ Yamato Tsuboi*, ${ }^{1,2}$ Shunsuke Murata, ${ }^{3}$ Fumihiro Naruse, ${ }^{1}$ Rei Ono. ${ }^{1}$ Kobe University Graduate School of Health Sciences, Department of Health Sciences; ${ }^{2}$ Japan Society for the Promotion of Science, Research Fellowship for Young Scientists; ${ }^{3}$ Every Rehab Inc

\subsection{6/oemed-2018-ICOHabstracts.750}

Introduction Presenteeism has an impact on socioeconomic burden. Low back pain (LBP) is also prevalent problem in eldercare workers and causes presenteeism. Kinesiophobia (fear of movement) is an important psychosocial factor because it is shown more disabling than pain itself. For resolving presenteeism, this study aimed to elucidate the association between kinesiophobia and presenteeism among eldercare workers with LBP.

Methods In this cross-sectional study, we identified 548 eldercare workers with LBP from the database collected in 2014. 343 participants were included for statistical analyses (median 48 years old, female $83.7 \%$ ). To measure kinesiophobia, we used the 11-item Tampa Scale for Kinesiophobia (TSK). TSK score ranges from 11 to 44, with higher score indicating higher kinesiophobia. 25-item Work Limitations Questionnaire (WLQ) was used to evaluate presenteeism and consisted of 'Time Management (TM)', 'Mental-Interpersonal Demands (MID)', 'Physical Demands (PD)', and 'Output Demands (OD)'. Productivity loss (\%) was estimated from WLQ using algorithm, and categorised into no $(<5 \%)$, mild $(5 \%$ to $10.9 \%)$, moderate $(11 \%$ to $16.9 \%)$, and severe presenteeism $(17 \%<)$. WLQ subscales were also categorised into quartile. For the univariate and multivariate analyses, ordinal logistic regression analyses were performed to test associations of TSK score with presenteeism. Covariates were demographic data, LBP status, lifestyle-related factors, and psychosocial factors. Proportional odds ratios (OR) and 95\% confidence intervals (95\% CI) were estimated.

Results In the univariate analysis, TSK score was significantly associated with productivity loss and all WLQ subscales. After adjusting for covariates, higher TSK score was significantly associated with larger productivity loss $(\mathrm{OR}=1.11$, 95\% CI: 1.06 to 1.17). Associations of TSK score with all WLQ subscales also remained significant after adjustment for covariates (TM; OR=1.05, 95\% CI: 1.01 to $1.09, \mathrm{MID} ; \mathrm{OR}=1.10$, 95\% CI: 1.05 to $1.15, \mathrm{PD}$; OR $=1.05,95 \% \mathrm{CI}$ : 1.00 to 1.09 , $\mathrm{OD} ; \mathrm{OR}=1.05,95 \% \mathrm{CI}: 1.01$ to 1.10 ).

Conclusion This study suggests that kinesiophobia could be an important factors related to presenteeism among eldercare workers with LBP.

\section{ERGONOMIC RISK FACTORS IN INTENSIVE CARE UNIT AND MUSCULOSKELETAL SYMPTOMS}

${ }^{1}$ Banu Dilek, ${ }^{2}$ Ayse Coskun Beyan, ${ }^{2}$ Sabriye Özcan, ${ }^{2}$ Tugba Demirel, ${ }^{2}$ Özay Işık, ${ }^{2}$ Yucel Demiral. 'Dokuz Eylul University Physical Therapy and Rehabilitation Department, Izmir, Turkey; ${ }^{2}$ Dokuz Eylul University Occupational Medicine Department, Izmir, Turkey

10.1136/oemed-2018-ICOHabstracts.751 